\title{
A Hybrid Method to Improve Forecasting Accuracy Utilizing Genetic Algorithm -An Application to the Data of Operating equipment and supplies
}

\author{
Daisuke Takeyasu \\ The Open University of Japan, 2-11 Wakaba, \\ Mihama-District, Chiba City, 261-8586, \\ Japan
}

\begin{abstract}
In industries, how to improve forecasting accuracy such as sales, shipping is an important issue. There are many researches made on this. In this paper, a hybrid method is introduced and plural methods are compared. Focusing that the equation of exponential smoothing method(ESM) is equivalent to $(1,1)$ order ARMA model equation, new method of estimation of smoothing constant in exponential smoothing method is proposed before by us which satisfies minimum variance of forecasting error. Generally, smoothing constant is selected arbitrarily. But in this paper, we utilize above stated theoretical solution. Firstly, we make estimation of ARMA model parameter and then estimate smoothing constants. Thus theoretical solution is derived in a simple way and it may be utilized in various fields. Furthermore, combining the trend removing method with this method, we aim to improve forecasting accuracy. An approach to this method is executed in the following method. Trend removing by the combination of linear and $2^{\text {nd }}$ order non-linear function and $3^{\text {rd }}$ order non-linear function is executed to the data of Operating equipment and supplies for three cases (An injection device and a puncture device, A sterilized hypodermic needle and A sterilized syringe). The weights for these functions are set 0.5 for two patterns at first and then varied by 0.01 increment for three patterns and optimal weights are searched. Genetic Algorithm is utilized to search the optimal weight for the weighting parameters of linear and non-linear function. For the comparison, monthly trend is removed after that. Theoretical solution of smoothing constant of ESM is calculated for both of the monthly trend removing data and the non-monthly trend removing data. Then forecasting is executed on these data. The new method shows that it is useful for the time series that has various trend characteristics and has rather strong seasonal trend. The effectiveness of this method should be examined in various cases.
\end{abstract}

Keywords-minimum variance; exponential smoothing method; forecasting; trend; operating equipment and supplies

\section{INTRODUCTION}

Time series analysis is often used in such themes as sales forecasting, stock market price forecasting etc. Sales forecasting is inevitable for Supply Chain Management. But in fact, it is not well utilized in industries. It is because there are so many irregular incidents therefore it becomes hard to make sales forecasting. A mere application of method does not bear good result. The big reason is that sales data or production data are not stationary time series, while linear model requires the

\author{
Kazuhiro Takeyasu $\uparrow$ \\ College of Business Administration, Tokoha \\ University,325 Oobuchi, Fuji City, Shizuoka, 417-0801, \\ Japan
}

time series as a stationary one. In order to improve forecasting accuracy, we have devised trend removal methods as well as searching optimal parameters and obtained good results. We created a new method and applied it to various time series and examined the effectiveness of the method. Applied data are sales data, production data, shipping data, stock market price data, flight passenger data etc.

Many methods for time series analysis have been presented such as Autoregressive model (AR Model), Autoregressive Moving Average Model (ARMA Model) and Exponential Smoothing Method $(\mathrm{ESM})^{[1]-[4]}$. Among these, ESM is said to be a practical simple method.

For this method, various improving method such as adding compensating item for time lag, coping with the time series with trend ${ }^{[5]}$, utilizing Kalman Filter ${ }^{[6]}$, Bayes Forecasting ${ }^{[7]}$, adaptive ESM $^{[8]}$, exponentially weighted Moving Averages with irregular updating periods ${ }^{[9]}$, making averages of forecasts using plural method ${ }^{[0]}$ are presented. For example, Maeda ${ }^{[6]}$ calculated smoothing constant in relationship with S/N ratio under the assumption that the observation noise was added to the system. But he had to calculate under supposed noise because he could not grasp observation noise. It can be said that it doesn't pursue optimum solution from the very data themselves which should be derived by those estimation.

Ishii ${ }^{[11]}$ pointed out that the optimal smoothing constant was the solution of infinite order equation, but he didn't show analytical solution. Based on these facts, we proposed a new method of estimation of smoothing constant in ESM before $[12],[20]$. Focusing that the equation of ESM is equivalent to $(1,1)$ order ARMA model equation, a new method of estimation of smoothing constant in ESM was derived. Furthermore, combining the trend removal method, forecasting accuracy was improved, where shipping data, stock market price data etc. were examined [13]-[20].

In this paper, utilizing above stated method, a revised forecasting method is proposed. In making forecast such as production data, trend removing method is devised. Trend removing by the combination of linear and $2^{\text {nd }}$ order non-linear function and $3^{\text {rd }}$ order non-linear function is executed to the data of Operating equipment and supplies for three cases (An injection device and a puncture device, A sterilized hypodermic needle and A sterilized syringe). These Operating 
equipment and supplies are used for medical use. The weights for these functions are set 0.5 for two patterns at first and then varied by 0.01 increments for three patterns and optimal weights are searched. Genetic Algorithm is utilized to search the optimal weight for the weighting parameters of linear and non-linear function. For the comparison, monthly trend is removed after that. Theoretical solution of smoothing constant of ESM is calculated for both of the monthly trend removing data and the non monthly trend removing data. Then forecasting is executed on these data. This is a revised forecasting method. Variance of forecasting error of this newly proposed method is assumed to be less than those of previously proposed method. The rest of the paper is organized as follows. In section 2, ESM is stated by ARMA model and estimation method of smoothing constant is derived using ARMA model identification. The combination of linear and non-linear function is introduced for trend removing in section 3. The Monthly Ratio is referred in section 4. Forecasting Accuracy is defined in section 5. Optimal weights are searched in section 6. Forecasting is carried out in section 7 , and estimation accuracy is examined.

\section{Description Of Esm Using ARma Model [12]}

In ESM, forecasting at time $t+1$ is stated in the following equation

$$
\begin{array}{r}
\hat{x}_{t+1}=\hat{x}_{t}+\alpha\left(x_{t}-\hat{x}_{t}\right) \\
=\alpha x_{t}+(1-\alpha) \hat{x}_{t}
\end{array}
$$

Here,

$$
\begin{gathered}
\hat{x}_{t+1} \text { : forecasting at } t+1 \\
x_{t}: \text { realized value at } t
\end{gathered}
$$$$
\alpha \text { : smoothing constant }(0<\alpha<1)
$$

(2) is re-stated as

$$
\hat{x}_{t+1}=\sum_{l=0}^{\infty} \alpha(1-\alpha)^{l} x_{t-l}
$$

By the way, we consider the following $(1,1)$ order ARMA model.

$$
x_{t}-x_{t-1}=e_{t}-\beta e_{t-1}
$$

Generally, $(p, q)$ order ARMA model is stated as

$$
x_{t}+\sum_{i=1}^{p} a_{i} x_{t-i}=e_{t}+\sum_{j=1}^{q} b_{j} e_{t-j}
$$

Here,

MA process in (5) is supposed to satisfy convertibility condition. Utilizing the relation that

$$
E\left[e_{t} \mid e_{t-1}, e_{t-2}, \cdots\right]=0
$$

we get the following equation from (4)

$$
\hat{x}_{t}=x_{t-1}-\beta e_{t-1}
$$

Operating this scheme on $t+1$, we finally get

$$
\begin{aligned}
\hat{x}_{t+1} & =\hat{x}_{t}+(1-\beta) e_{t} \\
& =\hat{x}_{t}+(1-\beta)\left(x_{t}-\hat{x}_{t}\right)
\end{aligned}
$$

If we set $1-\beta=\alpha$, the above equation is the same with (1), i.e., equation of ESM is equivalent to $(1,1)$ order ARMA model, or is said to be $(0,1,1)$ order ARIMA model because $1 \mathrm{st}$ order AR parameter is -1 . Comparing with (4) and (5), we obtain

$$
\left\{\begin{array}{l}
a_{1}=-1 \\
b_{1}=-\beta
\end{array}\right.
$$

From (1), (7),

$$
\alpha=1-\beta
$$

Therefore, we get

$$
\left\{\begin{array}{l}
a_{1}=-1 \\
b_{1}=-\beta=\alpha-1
\end{array}\right.
$$

From above, we can get estimation of smoothing constant after we identify the parameter of MA part of ARMA model. But, generally MA part of ARMA model become non-linear equations which are described below.

Let (5) be

$$
\begin{aligned}
& \tilde{x}_{t}=x_{t}+\sum_{i=1}^{p} a_{i} x_{t-i} \\
& \tilde{x}_{t}=e_{t}+\sum_{j=1}^{q} b_{j} e_{t-j}
\end{aligned}
$$

We express the autocorrelation function of $\tilde{x}_{t}$ as $\tilde{r}_{k}$ and from (9), (10), we get the following non-linear equations which are well known.

$$
\begin{aligned}
& \left\{x_{t}\right\} \text { : Sample process of Stationary Ergodic Gaussian } \\
& \text { Process } x(t) t=1,2, \cdots, N, \cdots \\
& \left\{e_{t}\right\} \text { : Gaussian White Noise with } 0 \text { mean } \sigma_{e}^{2} \text { variance }
\end{aligned}
$$




$$
\left\{\begin{array}{lr}
\tilde{r}_{k}=\sigma_{e}^{2} \sum_{j=0}^{q-k} b_{j} b_{k+j} & (k \leq q) \\
0 & (k \geq q+1) \\
\tilde{r}_{0}=\sigma_{e}^{2} \sum_{j=0}^{q} b_{j}^{2} &
\end{array}\right\}
$$

For these equations, recursive algorithm has been developed. In this paper, parameter to be estimated is only $b_{1}$, so it can be solved in the following way.

From (4) (5) (8) (11), we get

$$
\begin{aligned}
& q=1 \\
& a_{1}=-1 \\
& b_{1}=-\beta=\alpha-1 \\
& \tilde{r}_{0}=\left(1+b_{1}^{2}\right) \sigma_{e}^{2} \\
& \tilde{r}_{1}=b_{1} \sigma_{e}^{2}
\end{aligned}
$$

If we set

$$
\rho_{k}=\frac{\tilde{r}_{k}}{\tilde{r}_{0}}
$$

the following equation is derived

$$
\rho_{1}=\frac{b_{1}}{1+b_{1}^{2}}
$$

We can get $b_{1}$ as follows

$$
b_{1}=\frac{1 \pm \sqrt{1-4 \rho_{1}^{2}}}{2 \rho_{1}}
$$

In order to have real roots, $\rho_{1}$ must satisfy

$$
\left|\rho_{1}\right| \leq \frac{1}{2}
$$

From invertibility condition, $b_{1}$ must satisfy

$$
\left|b_{1}\right|<1
$$

From (14), using the next relation,

$$
\begin{aligned}
& \left(1-b_{1}\right)^{2} \geq 0 \\
& \left(1+b_{1}\right)^{2} \geq 0
\end{aligned}
$$

(16) always holds

As

$$
\alpha=b_{1}+1
$$

$b_{1}$ is within the range of

$$
-1<b_{1}<0
$$

Finally we get

$$
\left.\begin{array}{l}
b_{1}=\frac{1-\sqrt{1-4 \rho_{1}^{2}}}{2 \rho_{1}} \\
\alpha=\frac{1+2 \rho_{1}-\sqrt{1-4 \rho_{1}^{2}}}{2 \rho_{1}}
\end{array}\right\}
$$

which satisfies above condition. Thus we can obtain a theoretical solution by a simple way. Focusing on the idea that the equation of ESM is equivalent to $(1,1)$ order ARMA model equation, we can estimate smoothing constant after estimating ARMA model parameter. It can be estimated only by calculating 0 th and 1 st order autocorrelation function.

\section{TREND REMOVAl METHOD}

As trend removal method, we describe the combination of linear and non-linear function.

[1] Linear function

We set

$$
y=a_{1} x+b_{1}
$$

as a linear function.

[2] Non-linear function

We set

$$
\begin{gathered}
y=a_{2} x^{2}+b_{2} x+c_{2} \\
y=a_{3} x^{3}+b_{3} x^{2}+c_{3} x+d_{3}
\end{gathered}
$$

as a 2 nd and a 3 rd order non-linear function. $\left(a_{2}, b_{2}, c_{2}\right)$ and $\left(a_{3}, b_{3}, c_{3}, d_{3}\right)$ are also parameters for a 2 nd and a 3 rd order non-linear functions which are estimated by using least square method.

[3] The combination of linear and non-linear function.

We set

$$
\begin{aligned}
& y=\alpha_{1}\left(a_{1} x+b_{1}\right)+\alpha_{2}\left(a_{2} x^{2}+b_{2} x+c_{2}\right) \\
& +\alpha_{3}\left(a_{3} x^{3}+b_{3} x^{2}+c_{3} x+d_{3}\right) \\
& 0 \leq \alpha_{1} \leq 1,0 \leq \alpha_{2} \leq 1,0 \leq \alpha_{3} \leq 1, \alpha_{1}+\alpha_{2}+\alpha_{3}=1
\end{aligned}
$$

as the combination linear and 2nd order non-linear and 3rd order non-linear function. Trend is removed by dividing the original data by (21). The optimal weighting parameter 
$\alpha_{1}, \alpha_{2}, \alpha_{3}$, are determined by utilizing GA. GA method is precisely described in section 6 .

\section{MONTHLY RATIO}

For example, if there is the monthly data of $\mathrm{L}$ years as stated bellow:

$$
\left\{x_{i j}\right\}(i=1, \cdots, L)(j=1, \cdots, 12)
$$

Where, $x_{i j} \in R$ in which $j$ means month and $i$ means year and $x_{i j}$ is a shipping data of $i$-th year, $j$-th month. Then, monthly ratio $\tilde{x}_{j}(j=1, \cdots, 12)$ is calculated as follows.

$$
\tilde{x}_{j}=\frac{\frac{1}{L} \sum_{i=1}^{L} x_{i j}}{\frac{1}{L} \cdot \frac{1}{12} \sum_{i=1}^{L} \sum_{j=1}^{12} x_{i j}}
$$

Monthly trend is removed by dividing the data by (23). Numerical examples both of monthly trend removal case and non-removal case are discussed in 7.

\section{FORECASTING ACCURACY}

Forecasting accuracy is measured by calculating the variance of the forecasting error. Variance of forecasting error is calculated by:

$$
\sigma_{\varepsilon}^{2}=\frac{1}{N-1} \sum_{i=1}^{N}\left(\varepsilon_{i}-\bar{\varepsilon}\right)^{2}
$$

Where, forecasting error is expressed as:

$$
\begin{gathered}
\varepsilon_{i}=\hat{x}_{i}-x_{i} \\
\bar{\varepsilon}=\frac{1}{N} \sum_{i=1}^{N} \varepsilon_{i}
\end{gathered}
$$

\section{Searching Optimal Weights Utilizing Ga}

\section{A. Definition of the problem}

We search $\alpha_{1}, \alpha_{2}, \alpha_{3}$ of (21) which minimizes (24) by utilizing GA. By (22), we only have to determine $\alpha_{1}$ and $\alpha_{2}$. $\sigma_{\varepsilon}^{2}((24))$ is a function of $\alpha_{1}$ and $\alpha_{2}$, therefore we express them as $\sigma_{\varepsilon}^{2}\left(\alpha_{1}, \alpha_{2}\right)$. Now, we pursue the following:

$$
\begin{aligned}
& \text { Minimize: } \sigma_{\varepsilon}^{2}\left(\alpha_{1}, \alpha_{2}\right) \\
& \text { subject to: } 0 \leq \alpha_{1} \leq 1,0 \leq \alpha_{2} \leq 1, \alpha_{1}+\alpha_{2} \leq 1
\end{aligned}
$$

We do not necessarily have to utilize GA for this problem which has small member of variables. Considering the possibility that variables increase when we use logistics curve etc in the near future, we want to ascertain the effectiveness of GA.

\section{B. The structure of the gene}

Gene is expressed by the binary system using $\{0,1\}$ bit. Domain of variable is $[0,1]$ from (22).

We suppose that variables take down to the second decimal place. As the length of domain of variable is $1-0=1$, seven bits are required to express variables. The binary bit strings <bit6, $\sim$,bit $0>$ is decoded to the $[0,1]$ domain real number by the following procedure. ${ }^{[21]}$

Procedure 1: Convert the binary number to the binarycoded decimal.

$$
\begin{aligned}
& \left(\left\langle\text { bit }_{6}, \text { bit }_{5}, \text { bit }_{4}, \text { bit }_{3}, \text { bit }_{2}, \text { bit }_{1}, \text { bit }_{0}\right\rangle\right)_{2} \\
& =\left(\sum_{i=0}^{6} \text { bit }_{i} 2^{i}\right)_{10} \\
& =X^{\prime}
\end{aligned}
$$

Procedure 2: Convert the binary-coded decimal to the real number.

The real number

\begin{tabular}{|c|c|c|c|c|c|c|c|c|}
\hline \multirow{3}{*}{$\begin{array}{c}\text { The } \\
\text { decimal } \\
\text { number }\end{array}$} & \multicolumn{7}{|c|}{ The binary number } & \multirow{3}{*}{$\begin{array}{c}\text { The } \\
\text { Corresponding } \\
\text { real number }\end{array}$} \\
\hline & \multicolumn{7}{|c|}{ Position of the bit } & \\
\hline & 6 & 5 & 4 & 3 & 2 & 1 & 0 & \\
\hline 0 & 0 & 0 & 0 & 0 & 0 & 0 & 0 & 0.00 \\
\hline 1 & 0 & 0 & 0 & 0 & 0 & 0 & 1 & 0.01 \\
\hline 2 & 0 & 0 & 0 & 0 & 0 & 1 & 0 & 0.02 \\
\hline 3 & 0 & 0 & 0 & 0 & 0 & 1 & 1 & 0.02 \\
\hline 4 & 0 & 0 & 0 & 0 & 1 & 0 & 0 & 0.03 \\
\hline 5 & 0 & 0 & 0 & 0 & 1 & 0 & 1 & 0.04 \\
\hline 6 & 0 & 0 & 0 & 0 & 1 & 1 & 0 & 0.05 \\
\hline 7 & 0 & 0 & 0 & 0 & 1 & 1 & 1 & 0.06 \\
\hline 8 & 0 & 0 & 0 & 1 & 0 & 0 & 0 & 0.06 \\
\hline$\cdots$ & & & & & & & & $\ldots$ \\
\hline 126 & 1 & 1 & 1 & 1 & 1 & 1 & 0 & 0.99 \\
\hline 127 & 1 & 1 & 1 & 1 & 1 & 1 & 1 & 1.00 \\
\hline
\end{tabular}

$=($ Left hand starting point of the domain $)$

$+X^{\prime}$ ((Right hand ending point of the

domain $\left.) /\left(2^{7}-1\right)\right)$

The decimal number, the binary number and the corresponding real number in the case of 7 bits are expressed in Table 6-1.

TABLE 6-1: CoRRESPONDING TABLE Of THE Decimal Number, THE BinARY NUMBER AND THE REAL NUMBER 
1 variable is expressed by 7 bits, therefore 2 variables needs 14 bits. The gene structure is exhibited in Table 6-2.

Table 6-2: The gene structure

\begin{tabular}{|c|c|c|c|c|c|c||c|c|c|c|c|c|c|}
\hline \multicolumn{10}{|c||}{$\alpha_{1}$} & \multicolumn{10}{c|}{$\alpha_{2}$} \\
\hline \multicolumn{10}{|c|}{ Position of the bit } \\
\hline 13 & 12 & 11 & 10 & 9 & 8 & 7 & 6 & 5 & 4 & 3 & 2 & 1 & 0 \\
\hline \hline $0-$ & $0-$ & $0-$ & $0-$ & $0-$ & $0-$ & $0-$ & $0-$ & $0-$ & $0-$ & $0-$ & $0-$ & $0-$ & $0-$ \\
1 & 1 & 1 & 1 & 1 & 1 & 1 & 1 & 1 & 1 & 1 & 1 & 1 & 1 \\
\hline
\end{tabular}

C. The flow of Algorithm

The flow of algorithm is exhibited in Figure 6-1.

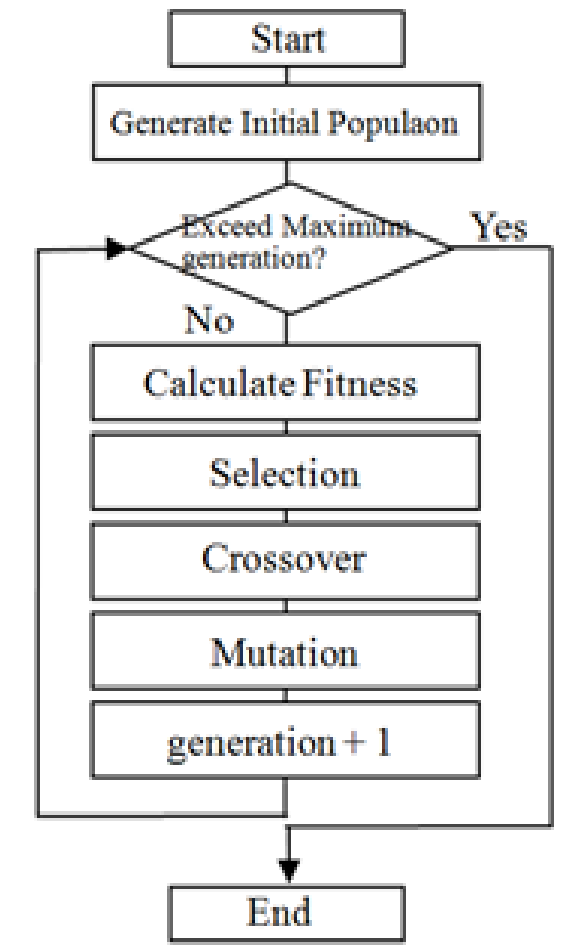

Figure 6-1: The flow of algorithm

\section{A. Initial Population}

Generate $M$ initial population. Here, $M=100$. Generate each individual so as to satisfy (22).

\section{B. Calculation of Fitness}

First of all, calculate forecasting value. There are 36 monthly data for each case. We use 24 data(1st to 24th) and remove trend by the method stated in section 3 . Then we calculate monthly ratio by the method stated in section 4 . After removing monthly trend, the method stated in section 2 is applied and Exponential Smoothing Constant with minimum variance of forecasting error is estimated.

Then 1 step forecast is executed. Thus, data is shifted to 2nd to 25th and the forecast for 26th data is executed consecutively, which finally reaches forecast of 36th data. To examine the accuracy of forecasting, variance of forecasting error is calculated for the data of 25 th to 36 th data. Final forecasting data is obtained by multiplying monthly ratio and trend. Variance of forecasting error is calculated by (24). Calculation of fitness is exhibited in Figure 6-2.

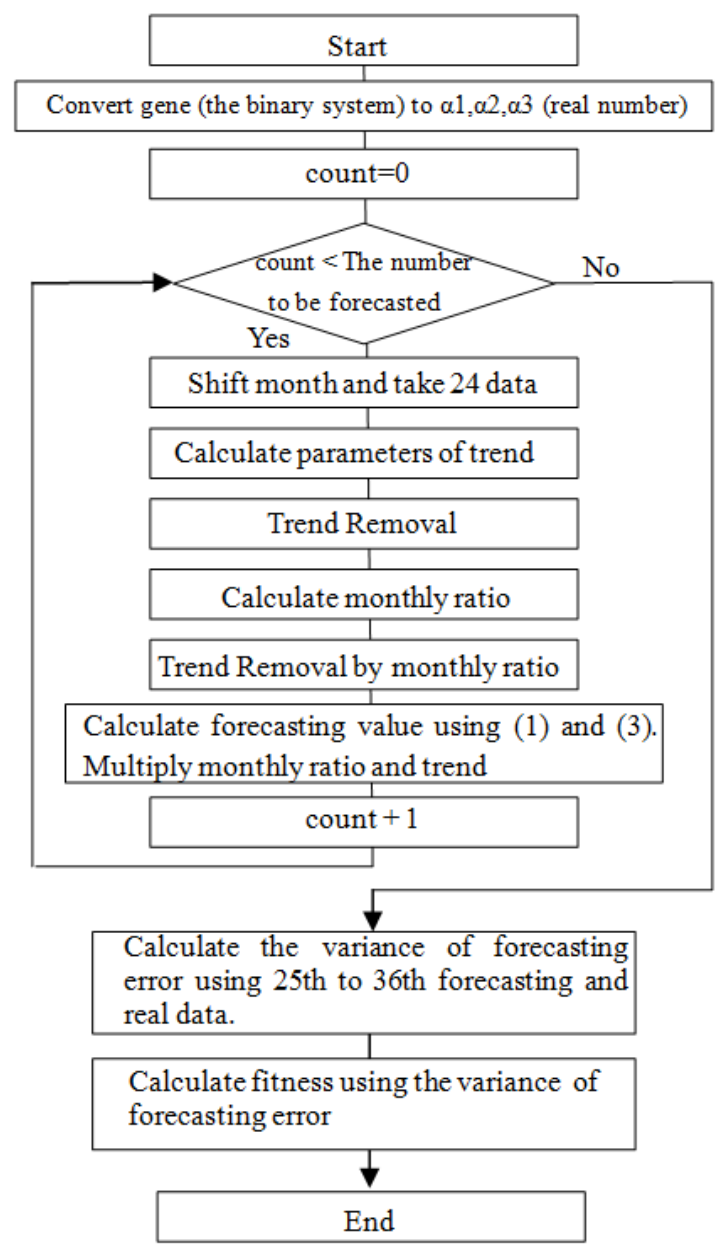

Figure 6-2: The flow of calculation of fitness

Scaling ${ }^{[22]}$ is executed such that fitness becomes large when the variance of forecasting error becomes small. Fitness is defined as follows

$$
f\left(\alpha_{1}, \alpha_{2}\right)=U-\sigma_{\varepsilon}^{2}\left(\alpha_{1}, \alpha_{2}\right)
$$

Where $U$ is the maximum of $\sigma_{\varepsilon}{ }^{2}\left(\alpha_{1}, \alpha_{2}\right)$ during the past $W$ generation. Here, $W$ is set to be 5 .

\section{Selection}

Selection is executed by the combination of the general elitist selection and the tournament selection. Elitism is executed until the number of new elites reaches the predetermined number. After that, tournament selection is executed and selected.

\section{Crossover}

Crossover is executed by the uniform crossover. Crossover rate is set as follows. 


$$
P_{c}=0.7
$$

\section{E. Mutation}

Mutation rate is set as follows

$$
P_{m}=0.05
$$

Mutation is executed to each bit at the probability $P_{m}$, therefore all mutated bits in the population $M$ becomes $P_{m} \times M \times 14$.

\section{NUMERICAL EXAMPLE}

\section{A. Application to the original production data of Wheelchairs}

The data of Operating equipment and supplies for three cases (An injection device and a puncture device, A sterilized hypodermic needle and A sterilized syringe) from January 2010 to December 2012 are analyzed. These data are obtained from the Annual Report of Statistical Investigation on Statistical-Survey-on-Trends-in-Pharmaceutical-Production by Ministry of Health, Labour and Welfare in Japan. Furthermore, GA results are compared with the calculation results of all considerable cases in order to confirm the effectiveness of GA approach. First of all, graphical charts of these time series data are exhibited in Figure 7-1 - 7-3.

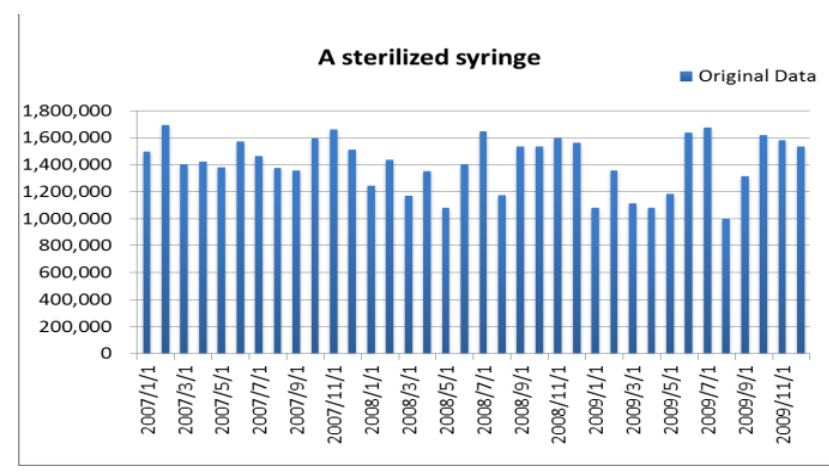

Figure 7-3: Domestic shipment data of a sterilized syringe

B. Execution Results

GA execution condition is exhibited in Table 7-1.

TABLE7-1: GA EXECUTION CONDITION GA execution condition

\begin{tabular}{|c|c|}
\hline \multicolumn{2}{|c|}{ GA execution condition } \\
\hline \hline Population & 100 \\
\hline Maximum Generation & 50 \\
\hline Crossover rate & 0.7 \\
\hline Mutation ratio & 0.05 \\
\hline Scaling window size & 5 \\
\hline The number of elites to retain & 2 \\
\hline Tournament size & 2 \\
\hline
\end{tabular}

We made 10 times repetition and the maximum, average, minimum of the variance of forecasting error and the average of convergence generation are exhibited in Table 7-2 and 7-3.

The variance of forecasting error for the case monthly ratio is not used is smaller than the case monthly ratio is used in A sterilized hypodermic needle. Other cases had good results in the case monthly ratio was used.

TABLE7-2: GA EXECUTION RESULTS (MONTHLY RATIO IS NOT USED)

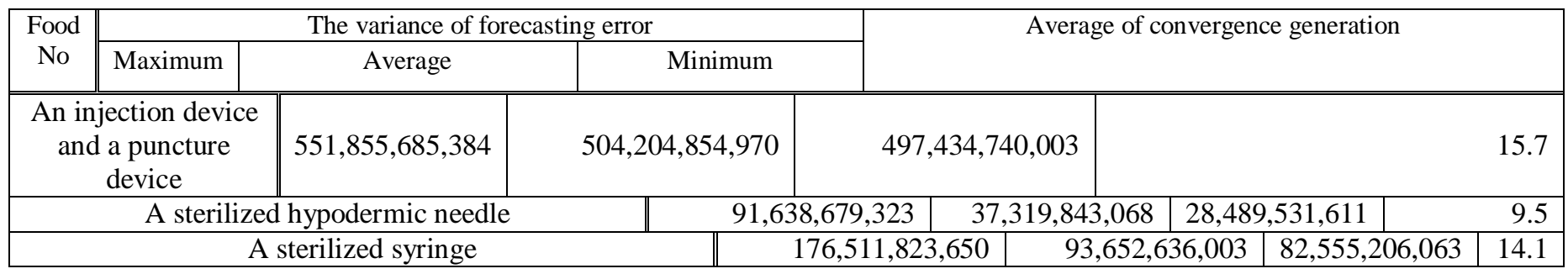

TABLE7-3: GA EXECUTION RESULTS (MONTHLY RATIO IS USED)

\begin{tabular}{|c||c|c|c|c|}
\hline \multirow{2}{*}{ Food No } & \multicolumn{2}{|c|}{ The variance of forecasting error } & $\begin{array}{c}\text { Average of } \\
\text { convergence } \\
\text { generation }\end{array}$ \\
\cline { 2 - 5 } & Maximum & Average & Minimum & 7.3 \\
\hline \hline $\begin{array}{c}\text { An injection device } \\
\text { and a puncture } \\
\text { device }\end{array}$ & $162,051,318,390$ & $106,546,694,680$ & $95,793,948,965$ & 11.1 \\
\hline $\begin{array}{c}\text { A sterilized } \\
\text { hypodermic needle }\end{array}$ & $79,422,467,024$ & $47,074,155,352$ & $42,493,594,397$ & 12.4 \\
\hline A sterilized syringe & $65,371,396,358$ & $38,622,248,849$ & $35,196,139,960$ & \\
\hline
\end{tabular}


The minimum variance of forecasting error of GA coincides with those of the calculation of all considerable cases and it shows the theoretical solution. Although it is a rather simple problem for GA, we can confirm the effectiveness of GA approach. Further study for complex problems should be examined hereafter.

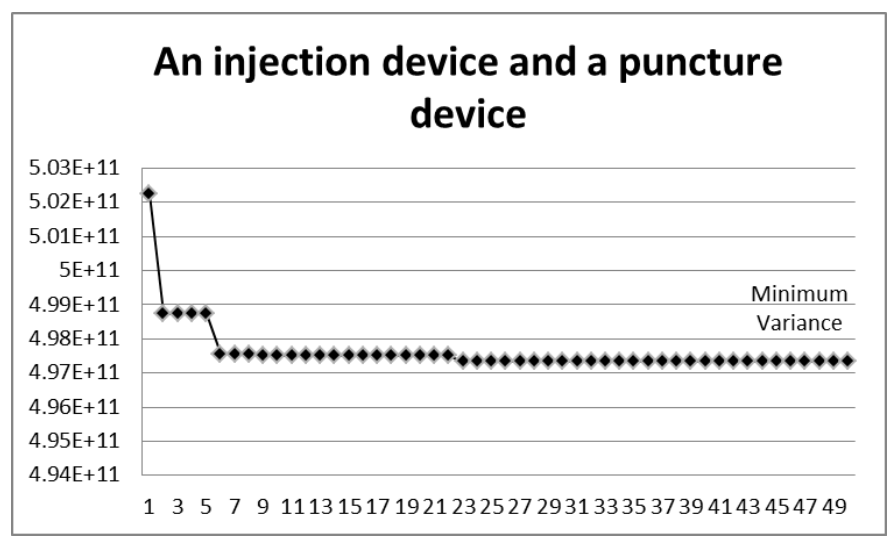

Figure7-4:Convergence Process in the case of An injection device and a puncture device (Monthly ratio is not used)

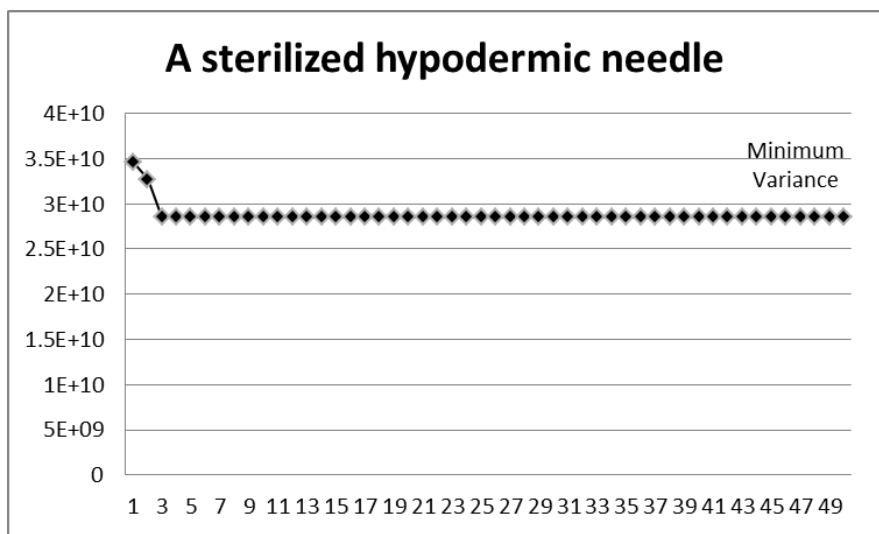

Figure 7-5:Convergence Process in the case of A sterilized hypodermic needle (Monthly ratio is used)

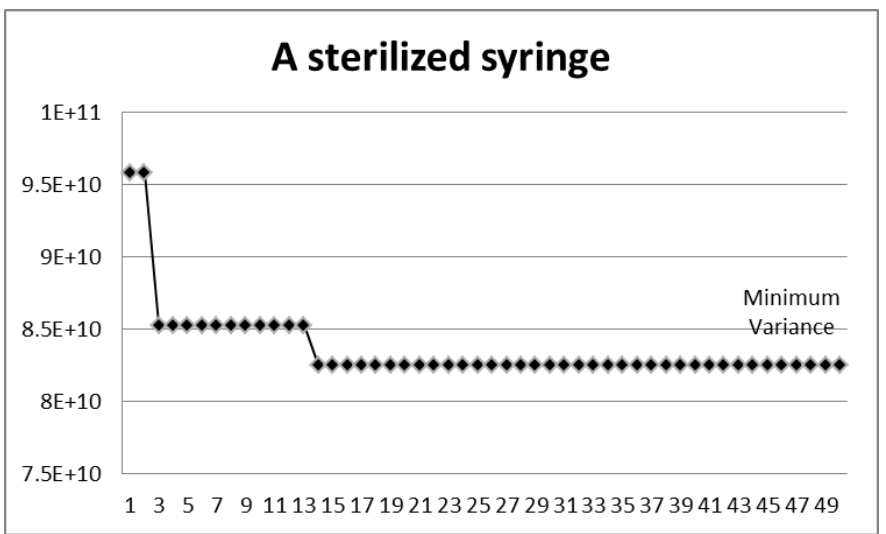

Figure7-6:Convergence Process in the case of A sterilized syringe (Monthly ratio is not used)

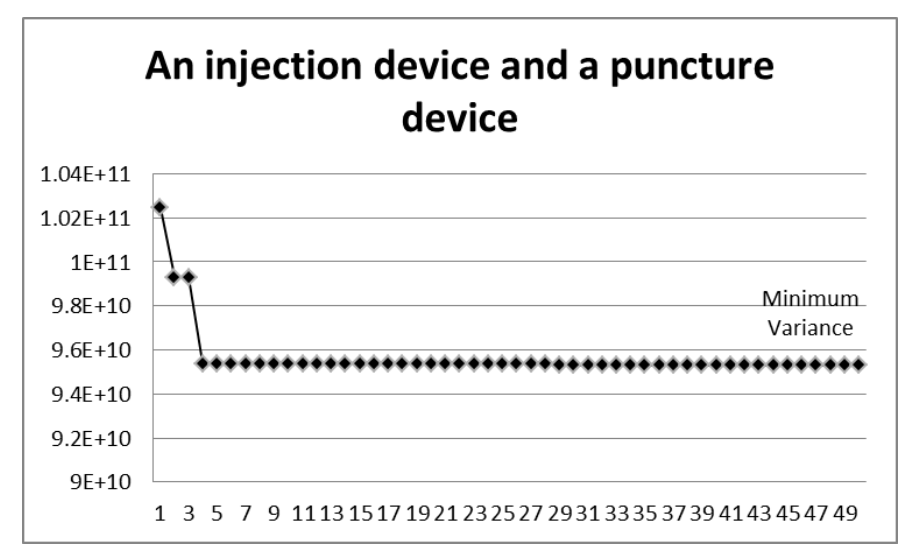

Figure7-7:Convergence Process in the case of An injection device and a puncture device

(Monthly ratio is used)

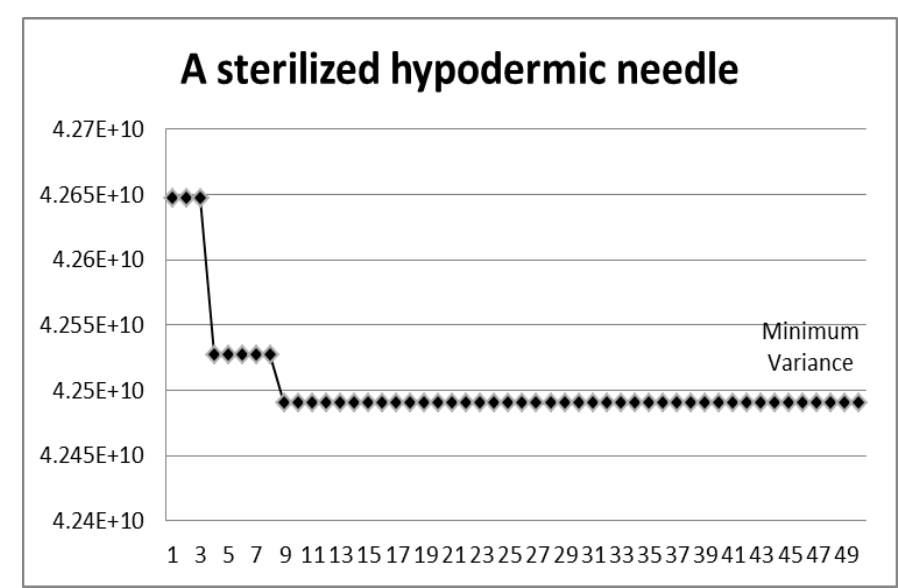

Figure 7-8: Convergence Process in the case of A sterilized hypodermic needle (Monthly ratio is not used)

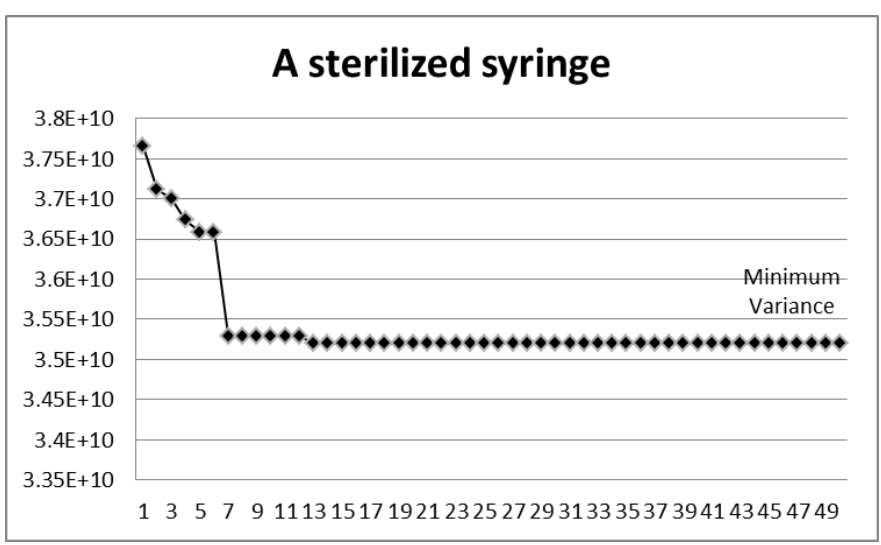

Figure7-9:Convergence Process in the case of A sterilized syringe (Monthly ratio is used)

Next, optimal weights and their genes are exhibited in Table 7-4,7-5. 
TABLE7-4: OPTIMAL WEIGHTS AND THEIR GENES (MONTHLY RATIO IS NOT USED)

\begin{tabular}{|c|c|c|c|c|c|c|c|c|c|c|c|c|c|c|c|c|c|}
\hline \multirow[t]{2}{*}{ Data } & \multirow[b]{2}{*}{$\alpha_{1}$} & \multirow[b]{2}{*}{$\alpha_{2}$} & \multirow[b]{2}{*}{$\alpha_{3}$} & \multicolumn{14}{|c|}{ position of the bit } \\
\hline & & & & 13 & 12 & 11 & 10 & 9 & 8 & 7 & 6 & 5 & 4 & 3 & 2 & 1 & 0 \\
\hline $\begin{array}{l}\text { An injection device } \\
\text { and a puncture device }\end{array}$ & 0.23 & 0.77 & 0 & 0 & 0 & 1 & 1 & 1 & 0 & 1 & 1 & 1 & 0 & 0 & 0 & 1 & 0 \\
\hline $\begin{array}{c}\text { A sterilized } \\
\text { hypodermic needle }\end{array}$ & 0.83 & 0.08 & 0.09 & 1 & 1 & 0 & 1 & 0 & 0 & 1 & 0 & 0 & 0 & 1 & 0 & 1 & 0 \\
\hline A sterilized syringe & 1.00 & 0 & 0 & 1 & 1 & 1 & 1 & 1 & 1 & 1 & 0 & 0 & 0 & 0 & 0 & 0 & 0 \\
\hline
\end{tabular}

TABLE 7-5: OPTIMAL WEIGHTS AND THEIR GENES (MONTHLY RATIO IS USED)

\begin{tabular}{|c|c|c|c|c|c|c|c|c|c|c|c|c|c|c|c|c|c|}
\hline \multirow[t]{2}{*}{ Data } & \multirow[b]{2}{*}{$\alpha_{1}$} & \multirow[b]{2}{*}{$\alpha_{2}$} & \multirow[b]{2}{*}{$\alpha_{3}$} & \multicolumn{14}{|c|}{ position of the bit } \\
\hline & & & & 13 & 12 & 11 & 10 & 9 & 8 & 7 & 6 & 5 & 4 & 3 & 2 & 1 & 0 \\
\hline $\begin{array}{l}\text { An injection device } \\
\text { and a puncture device }\end{array}$ & 0.02 & 0.98 & 0 & 0 & 0 & 0 & 0 & 0 & 1 & 0 & 1 & 1 & 1 & 1 & 1 & 0 & 1 \\
\hline $\begin{array}{c}\text { A sterilized } \\
\text { hypodermic needle }\end{array}$ & 0 & 0.37 & 0.63 & 0 & 0 & 0 & 0 & 0 & 0 & 0 & 0 & 1 & 0 & 1 & 1 & 1 & 1 \\
\hline A sterilized syringe & 1.00 & 0 & 0 & 1 & 1 & 1 & 1 & 1 & 1 & 1 & 0 & 0 & 0 & 0 & 0 & 0 & 0 \\
\hline
\end{tabular}

The linear function model is best in A sterilized syringe. An injection device and a puncture device selected $1^{\text {st }}+2^{\text {nd }}$ order function as the best one. A sterilized hypodermic needle selected $1^{\text {st }}+2^{\text {nd }}+3^{\text {rd }}$ order function as the best one.
These results were same for both of "Monthly ratio is not used" and "Monthly ratio is not used". Parameter estimation results for the trend of equation (21) using least square method are exhibited in Table 7-6 for the case of 1st to 24th data.Trend curves are exhibited in Figure 7-10 - 7-12.

TABLE 7-6: PARAMETER ESTIMATION RESULTS FOR THE TREND OF EQUATION (21)

\begin{tabular}{|c|c|c||c|c|c|c|c|c|c|}
\hline Data & $a_{1}$ & $b_{1}$ & $a_{2}$ & $b_{2}$ & $c_{2}$ & $a_{3}$ & $b_{3}$ & $c_{3}$ & $d_{3}$ \\
\hline \hline $\begin{array}{c}\text { An injection } \\
\text { device and a } \\
\text { puncture device }\end{array}$ & 8836.40 & 3971839.34 & 3616.96 & - & 4363676.73 & 466.59 & - & 96978.10 & 3954240.07 \\
\hline $\begin{array}{c}\text { A sterilized } \\
\text { hypodermic needle }\end{array}$ & 7625.06 & 717466.59 & 369.11 & -1602.74 & 757453.7 & 242.23 & -8714.55 & 91099.12 & 544895.87 \\
\hline $\begin{array}{c}\text { A sterilized } \\
\text { syringe }\end{array}$ & 2041.09 & 1469255.56 & 1353.07 & 35867.75 & -1615837.78 & 161.02 & -4685.33 & 25756.08 & 1474539.34 \\
\hline
\end{tabular}




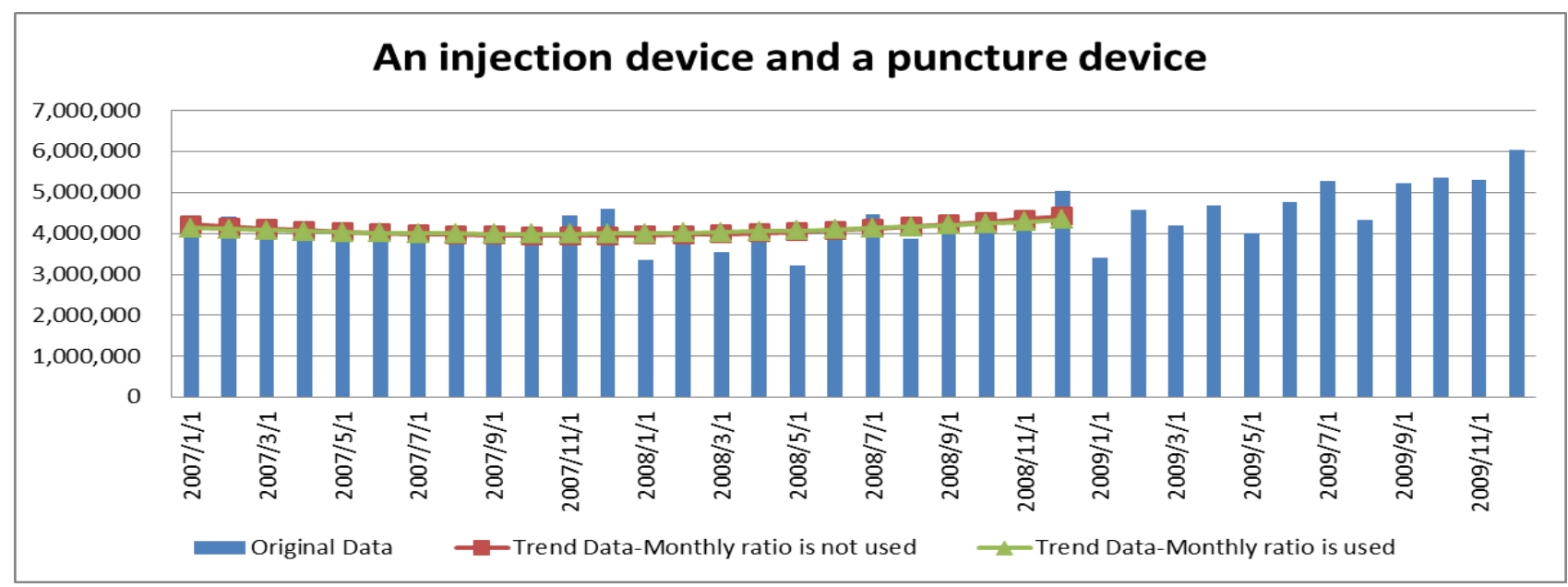

Figure7-10: Trend of An injection device and a puncture device

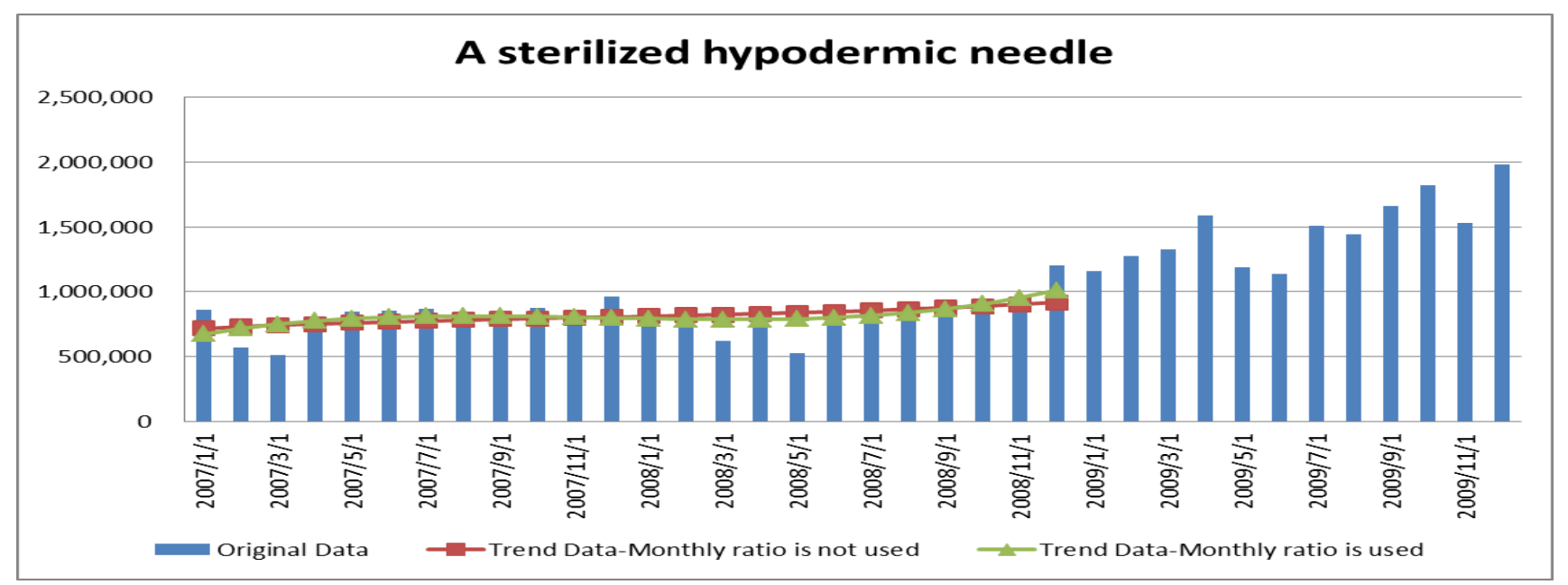

Figure7-11: Trend of A sterilized hypodermic needle 


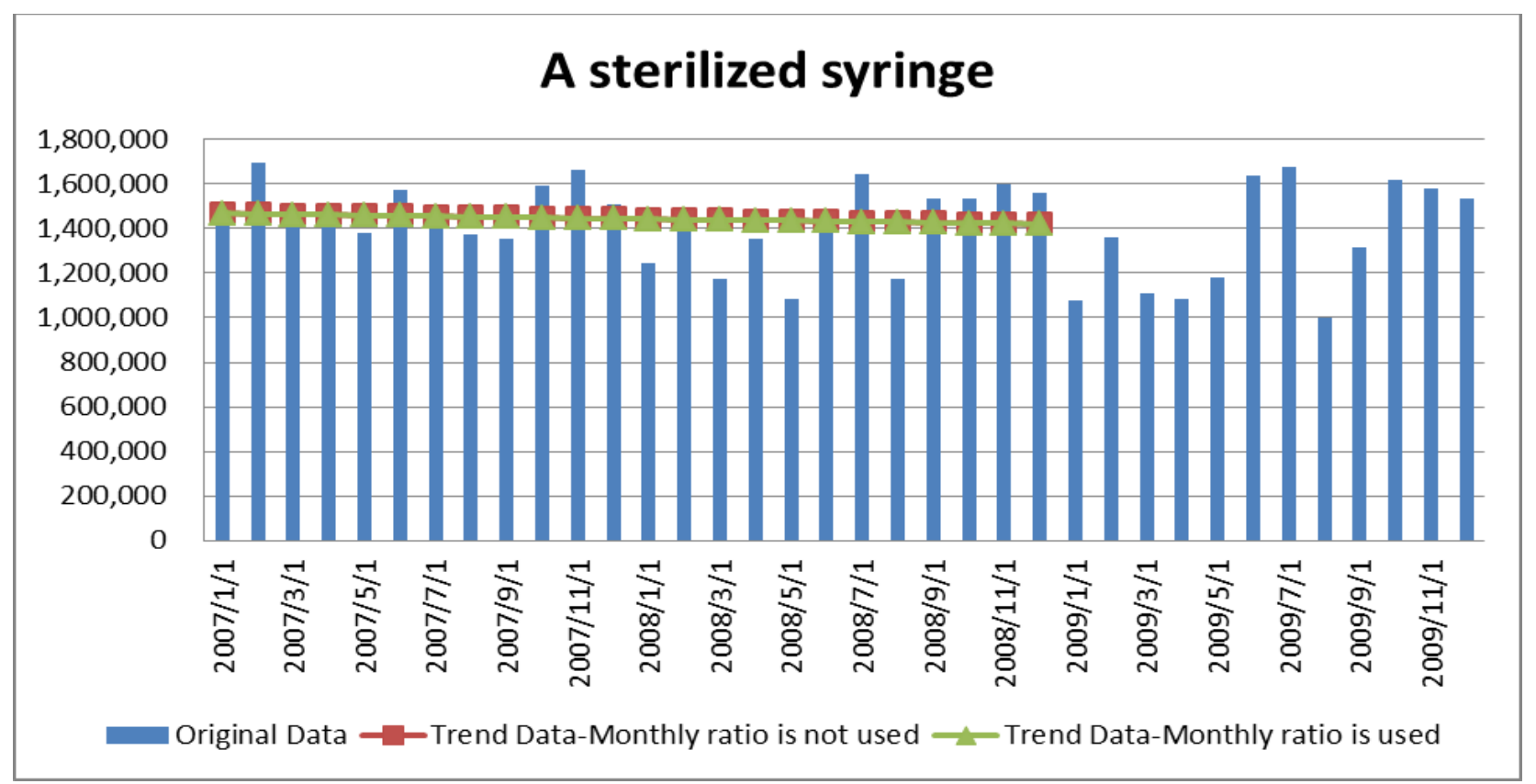

Figure7-12: Trend of A sterilized syringe

Calculation results of Monthly ratio for 1 st to 24 th data are exhibited in Table 7-7.

Table 7-7: PARAMETER ESTIMATION RESULT OF MONTHLY RATIO

\begin{tabular}{|c||c|c|c|c|c|c|c|c|c|c|c|c|}
\hline Date. & 1 & 2 & 3 & 4 & 5 & 6 & 7 & 8 & 9 & 10 & 11 & 12 \\
\hline \hline $\begin{array}{c}\text { An injection device and } \\
\text { a puncture device }\end{array}$ & 0.901 & 1.038 & 0.960 & 1.015 & 0.868 & 0.997 & 1.039 & 0.968 & 0.999 & 0.995 & 1.063 & 1.158 \\
\hline $\begin{array}{c}\text { A sterilized hypodermic } \\
\text { needle }\end{array}$ & 1.146 & 0.900 & 0.735 & 1.072 & 0.866 & 1.033 & 1.094 & 0.984 & 0.993 & 0.993 & 0.986 & 1.198 \\
\hline A sterilized syringe & 0.941 & 1.076 & 0.887 & 0.95 & 0.84 & 1.02 & 1.078 & 0.883 & 1.004 & 1.088 & 1.137 & 1.071 \\
\hline
\end{tabular}

Estimation result of the smoothing constant of minimum variance for the 1st to 24th data are exhibited in Table 7-8, 7-9.

Forecasting results are exhibited in Table 7-13 - 7-15.

Table 7-8:SMOOTHING CONSTANT OF MINIMUM VARIANCE OF EQUATION (17) (MONTHLY RATIO IS NOT USED)

\begin{tabular}{|c||c|c|}
\hline Date & $\rho 1$ & $\alpha$ \\
\hline \hline $\begin{array}{c}\text { An injection device and a } \\
\text { puncture device }\end{array}$ & -0.147880 & 0.848737 \\
\hline $\begin{array}{c}\text { A sterilized hypodermic } \\
\text { needle }\end{array}$ & -0.342724 & 0.603356 \\
\hline A sterilized syringe & -0.499464 & 0.045270 \\
\hline
\end{tabular}

Table 7-9: SMOOTHING CONSTANT OF MINIMUM VARIANCE OF EQUATION (17) (MONTHLY RATIO IS USED)

\begin{tabular}{|c||c|c|}
\hline Date, & $\rho 1$ & $\alpha$ \\
\hline \hline $\begin{array}{c}\text { An injection device and a } \\
\text { puncture device }\end{array}$ & -0.293350 & 0.675822 \\
\hline $\begin{array}{c}\text { A sterilized hypodermic } \\
\text { needle }\end{array}$ & -0.067694 & 0.931993 \\
\hline A sterilized syringe & -0.171119 & 0.823554 \\
\hline
\end{tabular}




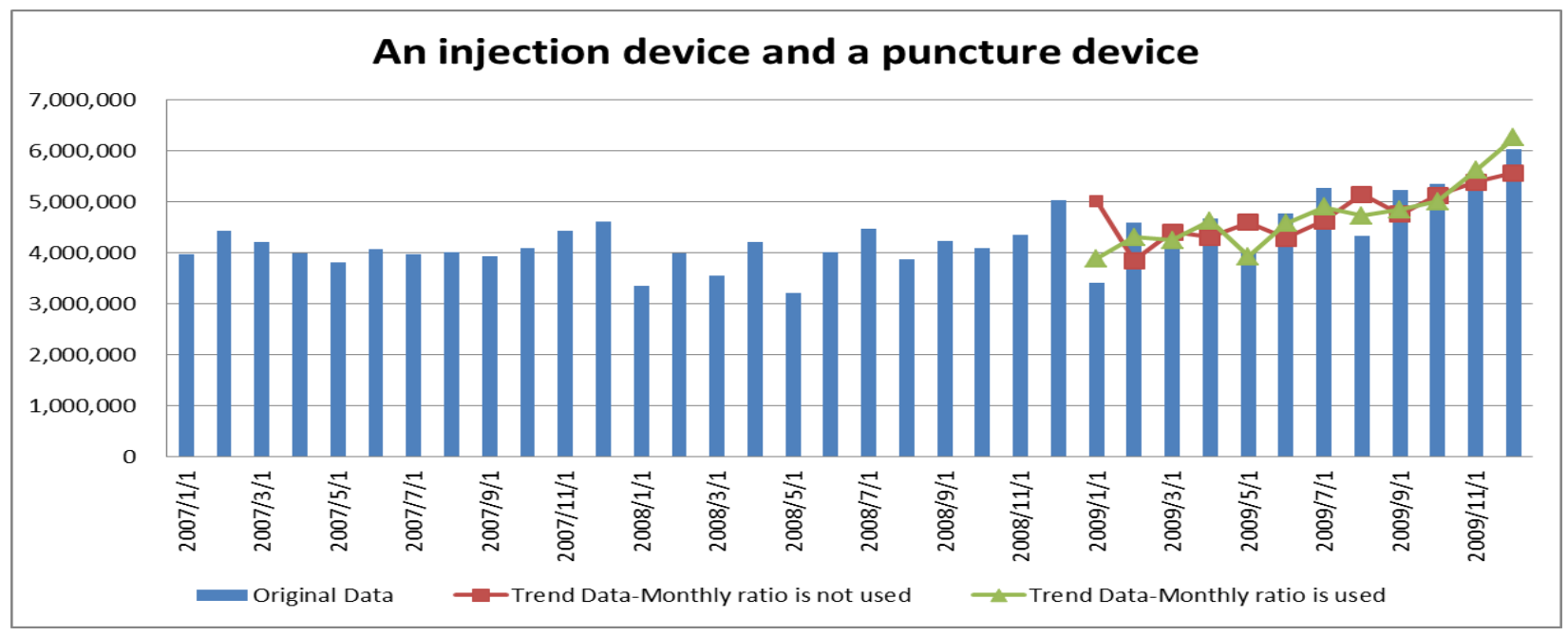

Figure 7-13: Forecasting Result of An injection device and a puncture device

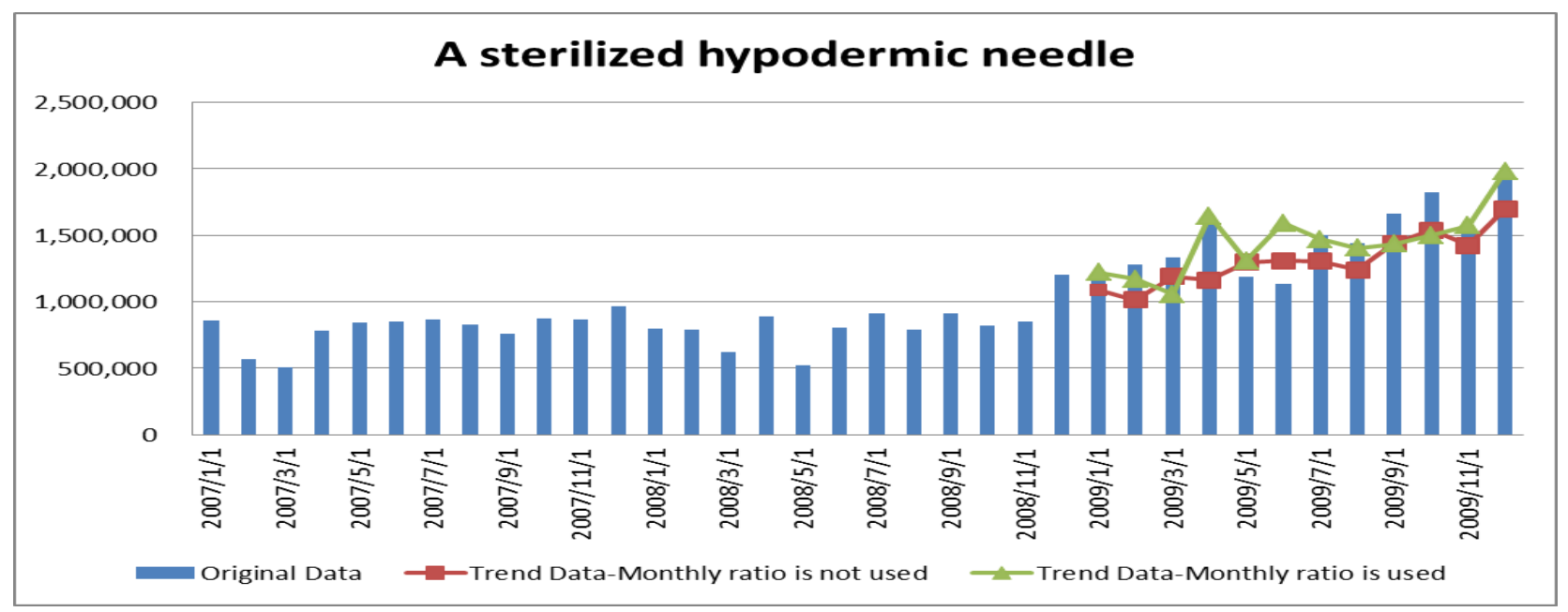

Figure 7-14: Forecasting Result of A sterilized hypodermic needle

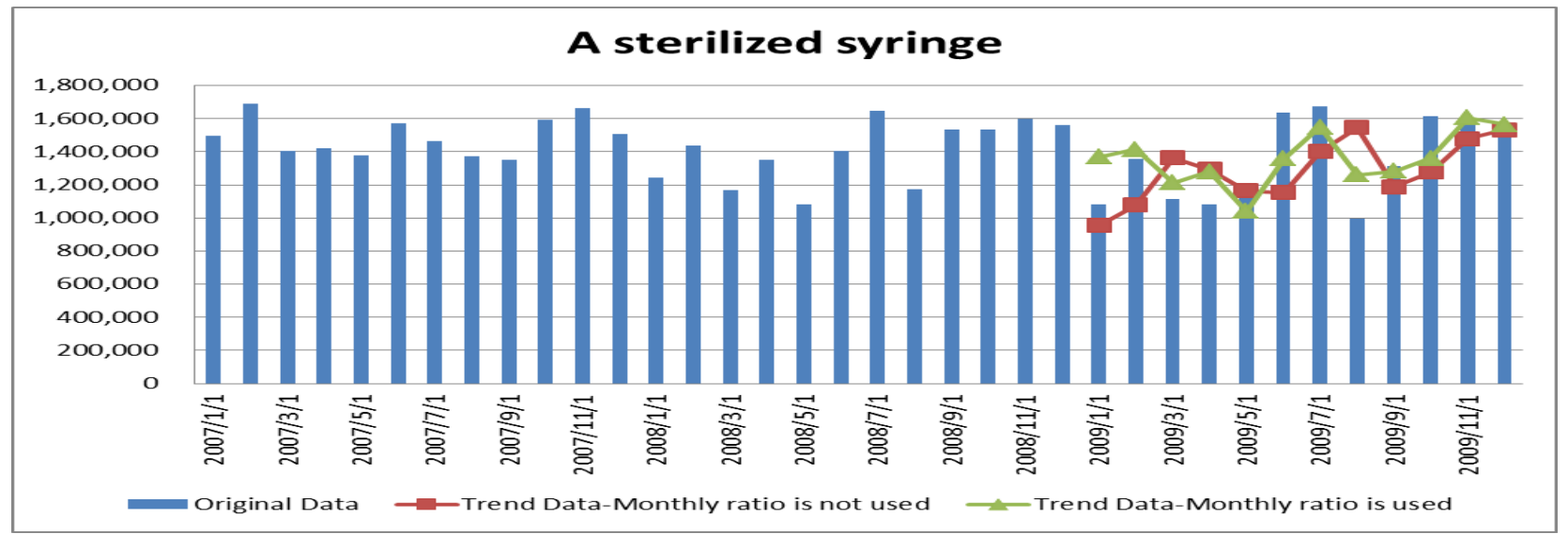

Figure 7-15: Forecasting Result of A sterilized syringe 


\section{Remarks}

The linear function model in the case Monthly ratio was used was best for $A$ sterilized syringe case. $1^{\text {st }}+2^{\text {nd }}$ function model in the case Monthly ratio was used was best for An injection device and a puncture device case. $1^{\text {st }}+2^{\text {nd }}+3^{\text {rd }}$ function model in the case Monthly ratio was not used was best for A sterilized hypodermic needle case.

The minimum variance of forecasting error of GA coincides with those of the calculation of all considerable cases and it shows the theoretical solution. Although it is a rather simple problem for GA, we can confirm the effectiveness of GA approach. Further study for complex problems should be examined hereafter.

\section{CONCLUSION}

Focusing on the idea that the equation of exponential smoothing method(ESM) was equivalent to $(1,1)$ order ARMA model equation, a new method of estimation of smoothing constant in exponential smoothing method was proposed before by us which satisfied minimum variance of forecasting error. Generally, smoothing constant was selected arbitrarily. But in this paper, we utilized above stated theoretical solution. Firstly, we made estimation of ARMA model parameter and then estimated smoothing constants. Thus theoretical solution was derived in a simple way and it might be utilized in various fields.

Furthermore, combining the trend removal method with this method, we aimed to improve forecasting accuracy. An approach to this method was executed in the following method. Trend removal by a linear function was applied to the data of Operating equipment and supplies for three cases (An injection device and a puncture device, A sterilized hypodermic needle and A sterilized syringe). The combination of linear and nonlinear function was also introduced in trend removal. Genetic Algorithm was utilized to search the optimal weight for the weighting parameters of linear and non-linear function. For the comparison, monthly trend was removed after that. Theoretical solution of smoothing constant of ESM was calculated for both of the monthly trend removing data and the non monthly trend removing data. Then forecasting was executed on these data. The new method shows that it is useful for the time series that has various trend characteristics. The effectiveness of this method should be examined in various cases.

\section{REFERENCES}

[1] Box Jenkins. (1994) Time Series Analysis Third Edition, Prentice Hall.

[2] R.G. Brown. (1963) Smoothing, Forecasting and Prediction of Discrete -Time Series, Prentice Hall.
[3] Hidekatsu Tokumaru et al. (1982) Analysis and Measurement - Theory and Application of Random data Handling, Baifukan Publishing.

[4] Kengo Kobayashi. (1992) Sales Forecasting for Budgeting, ChuokeizaiSha Publishing.

[5] Peter R.Winters. (1984) Forecasting Sales by Exponentially Weighted Moving Averages, Management Science, Vol6, No.3, pp. 324-343.

[6] Katsuro Maeda. (1984) Smoothing Constant of Exponential Smoothing Method, Seikei University Report Faculty of Engineering, No.38, pp. 2477-2484.

[7] M.West and P.J.Harrison. (1989) Baysian Forecasting and Dynamic Models, Springer-Verlag, New York.

[8] Steinar Ekern. (1982) Adaptive Exponential Smoothing Revisited, Journal of the Operational Research Society, Vol. 32 pp. 775-782.

[9] F.R.Johnston. (1993) Exponentially Weighted Moving Average (EWMA) with Irregular Updating Periods, Journal of the Operational Research Society, Vol.44, No.7 pp. 711-716.

[10] Spyros Makridakis and Robeat L.Winkler. (1983) Averages of Forecasts ; Some Empirical Results, Management Science, Vol.29, No.9, pp. 987-996.

[11] Naohiro Ishii et al. (1991) Bilateral Exponential Smoothing of Time Series, Int.J.System Sci., Vol.12, No.8, pp. 997-988.

[12] Kazuhiro Takeyasu and Keiko Nagata.(2010) Estimation of Smoothing Constant of Minimum Variance with Optimal Parameters of Weight, International Journal of Computational Science Vol.4,No.5, pp. 411-425.

[13] Kazuhiro Takeyasu, Keiko Nagata, Yuki Higuchi. (2009) Estimation of Smoothing Constant of Minimum Variance And Its Application to Shipping Data With Trend Removal Method, Industrial Engineering \& Management Systems (IEMS),Vol.8,No.4, pp.257-263,

[14] Kazuhiro Takeyasu, Keiko Nagata, Yui Nishisako. (2010) A Hybrid Method to Improve Forecasting Accuracy Utilizing Genetic Algorithm And Its Application to Industrial Data, NCSP'10, Honolulu,Hawaii,USA

[15] Kazuhiro Takeyasu, Keiko Nagata, Kana Takagi. (2010) Estimation of Smoothing Constant of Minimum Variance with Optimal Parameters of Weight, NCSP'10, Honolulu,Hawaii,USA

[16] Kazuhiro Takeyasu, Keiko Nagata, Tomoka Kuwahara. (2010) Estimation of Smoothing Constant of Minimum Variance Searching Optimal Parameters of Weight, NCSP'10, Honolulu,Hawaii,USA

[17] Kazuhiro Takeyasu, Keiko Nagata, Mai Ito, Yuki Higuchi (2010). A Hybrid Method to Improve Forecasting Accuracy Utilizing Genetic Algorithm, The 11th APEIMS, Melaka, Malaysia

[18] Kazuhiro Takeyasu, Keiko Nagata, Kaori Matsumura. (2011) Estimation of Smoothing Constant of Minimum Variance and Its Application to Sales Data, JAIMS, Honolulu, Hawaii, USA

[19] Hiromasa Takeyasu, Yuki Higuchi, Kazuhiro Takeyasu. (2012) A Hybrid Method to Improve Forecasting Accuracy in the Case of Bread, International Journal of Information and Communication Technology Research, Vol.2, No.11, pp.804 812.

[20] Kazuhiro Takeyasu and Kazuko Nagao.(2008) Estimation of Smoothing Constant of Minimum Variance and its Application to Industrial Data, Industrial Engineering and Management Systems, vol.7, no. 1, pp. 44-50.

[21] Masatosi Sakawa. Masahiro Tanaka. (1995) Genetic Algorithm 、 Asakura Pulishing Co., Ltd.

[22] Hitoshi Iba. (2002) Genetic Algorithm、Igaku Publishing. 